\title{
Role of Magnetic Resonance Imaging in Study of the Patellofemoral Joint Morphological Abnormalities Predisposing to Patellar Instability
}

\author{
H.P Parekh ${ }^{1}$, S Aravind Prasad Naik ${ }^{2}$ \\ ${ }^{1}$ Professor \& Head of Department, ${ }^{2} 3^{\text {rd }}$ Year Resident, \\ Department of Radio-Diagnosis, Shri M.P Shah Medical College, Jamnagar, India \\ Corresponding Author: S Aravind Prasad Naik
}

\begin{abstract}
Objectives: The Patellofemoral joint (PFJ) is a complex joint structure with high functional and biomechanical requirements. The purpose of this study is to evaluate cause of patella-femoral joint instability with in-depth evaluation of influence of anatomical risk factors (AF), measurements and anatomical relationships of the patellofemoral joint and various pathologies causing medial patellofemoral ligament (MPFL) injury that contributes to the patellar maltracking using 1.5 Tesla magnetic resonance imaging.
\end{abstract}

Materials and Methods: The present prospective study is carried out at department of radiology, Shri M P Shah Medical College, Jamnagar. This is study of total 50 patients carried out in 12-month duration from July 2019 to June 2020 , in which 50 patients aged between 8 - 68 years selected as study group presented with complaint symptoms of patellofemoral instability, knee pain and maltracking were examined and identified by an orthopaedic surgeon. Patients with the clinical suspicion of a PFJ maltracking were selected for subsequent MRI examination.

Results: The age distribution in our study was in range of 8 years to 68 years with maximum population within 31 to 40 years range, $66 \%$ were male and $34 \%$ female. Their trochlear depth, sulcal angle, trochlear facet asymmetry, lateral inclination angle was found abnormal in 18 cases $(36 \%), 8(16 \%)$ showed patellar alta and $7(14 \%)$ patella baja. Lateralization of patella (> $6 \mathrm{~mm}$ ) was noted in 18 patients (36\%), Medial patellofemoral ligament injuries were seen in $16(32 \%)$ patients on MRI and kissing contusion 10 (20\%) patients. A detailed understanding of the anatomy and pathology of the anterior knee compartment is essential for proper imaging interpretation and treatment management.

Conclusion: Magnetic resonance (MR) imaging has been established as the method of choice, showing high accuracy in patellofemoral joint abnormalities. The present study aims to explore the role of MR imaging in diagnosing the various disorders located in anterior compartment of knee.

Key words: Joint instability, Patellofemoral joint, MPFL, MR imaging/diagnosis.

\section{INTRODUCTION}

The knee joint is the largest joint of the human body. It is a modified hinge joint made up of two individual componentstibio-femoral and Patellofemoral joints. $(1,2)$ The Patellofemoral joint (PFJ) is a complex joint structure with high functional and biomechanical requirements. (3) the normal functioning of this joint (extension of the knee) is dependent on the congruent relationship of the patella with the trochlear groove.

Detailed study of the anatomy of the knee is fundamental for understanding the infirmities that affect it. Instability of the extensor apparatus, represented by recurrent dislocation and subluxation of the patella (4). This instability may affect both athletes and non-athletic individuals, causing pain and incapacity. It involves great 
biomechanical complexity and frequently necessitates surgical intervention to treat it.

It occurs most frequently in individuals with one or more anatomical abnormalities that predispose towards dislocation or subluxation, such as trochlear or patellar dysplasia, high patella, rotational deviations of the lower limbs, muscle dysplasia and lateral displacement of the patella by laxity/injury of the MPFL ligaments (5) Characterization and quantification of these anatomic anomalies will reveal the individual mechanism of patellar instability and help the orthopedic surgeon choose the optimal treatment.

MRI has now been accepted as the best modality for non -invasive radiation free imaging with multiplanar capabilities in the evaluation of knee pathologies (6).

The purpose of this article is to review the typical MR imaging findings in the knee after dislocation of the patella, including predisposing factors.

This is study of total 50 patients, aged between 8 - 68 years selected as study group referred to department of radiology from orthopaedic department were examined by an orthopaedic surgeon and Patients with the clinical suspicion of a PFJ maltracking were selected for subsequent MRI knee examination.

\section{METHODS}

The prospective study was conducted at Department of radio diagnosis, Shri Guru Gobind Singh Hospital and Shri M.P Shah Medical College, Jamnagar, Gujarat during July 2019 to June 2020. After taking informed consent, total 50 patients, aged between 8 to 68 years selected as study group referred to department of radiology from orthopaedic department were examined by an orthopaedic surgeon and Patients with the clinical suspicion of a PFJ maltracking were selected for subsequent MRI knee examination.

\section{Inclusion criteria}

The study included all patients with suspicion of or clinically diagnosed with extensor compartment abnormality of knee joint referred to department of radiology from orthopaedic department were examined by an orthopaedic surgeon.

\section{Exclusion criteria}

- Patient with any patient with absolute contraindications to MRI examination as cardiac pace maker, aneurysmal clipping, cochlear implants, hearing aids, intracranial aneurysmal clips and early pregnant patient.

- Patients having history of claustrophobia.

- Post treatment patients.

\section{Equipment used}

MRI: $\quad 1.5$ Tesla Magnetic Resonance Imaging system (Magnetom Essenza, Siemens health care, Germany)

\section{Technique}

Standard MRI of the knee with dedicated knee coil at our institution included sagittal T1 weighted TSE (TR/TE(ms)- 560/17, slice thickness- 3mm), Axial T1WI TSE (TR/TE(ms)- 580/14, slice thickness- $3 \mathrm{~mm}$ ), axial or coronal or sagittal fat suppressed PD sequence (TR/TE(ms)4500/30, slice thickness- $3 \mathrm{~mm})$, coronal T2WI (TR/TE(ms)- 3912/30, slice thickness- $3 \mathrm{~mm}$ ) and axial T1-weighted FSE post contrast (in a selected patient) and rarely sagittal PD (TR/TE(ms)- 4500/30, slice thickness- $3 \mathrm{~mm}$ )sequences.

\section{Technical description}

Patients were asked to remove metals, artificial prosthesis and taken to MRI room and were asked to sleep in supine position, patients undergoing MRI examination supine, feet first, knee coil (wraparound), place knee in the bottom half of the position comfort cushions at any pressure points, attach the top half of the coil and lock it in place. The need to restrict body movement during the scan time was explained to the patient. Then patient was 
H.P Parekh et.al. Role of magnetic resonance imaging in study of the patellofemoral joint morphological abnormalities predisposing to patellar instability

passed into MRI gentry, and patients details were entered in the console and standard knee sequences were taken.

Following parameters were assessed: Patellar height ratio, Lateral trochlear inclination angle, Sulcal angle, trochlear facet asymmetry, trochlear depth and Lateral patellofemoral angle, Lateralization of patella and Tibial tubercle to trochlear groove distance.

\section{Statistical Methods}

Descriptive analysis was carried out by the mean and standard deviation for quantitative variables, frequency and proportion for categorical s variables. Data was also represented using appropriate diagrams like a bar diagram $\mathrm{V}$, pie diagram and box plots.

\section{OBSERVATIONS AND RESULTS}

Table 1: Descriptive analysis of age-wise distribution in study population $(\mathrm{N}=50)$

\begin{tabular}{|c|c|c|}
\hline Age Group (Yrs) & No. Of Patients & Percentage \\
\hline $0-10$ & 1 & $2.0 \%$ \\
\hline $11-20$ & 4 & $8.0 \%$ \\
\hline $21-30$ & 10 & $20.0 \%$ \\
\hline $31-40$ & 16 & $32.0 \%$ \\
\hline $41-50$ & 9 & $18.0 \%$ \\
\hline $51-60$ & 6 & $12.0 \%$ \\
\hline$>61$ & 4 & $8.0 \%$ \\
\hline Total & \multicolumn{2}{|c|}{$\mathbf{2 0 \%}$} \\
\hline
\end{tabular}

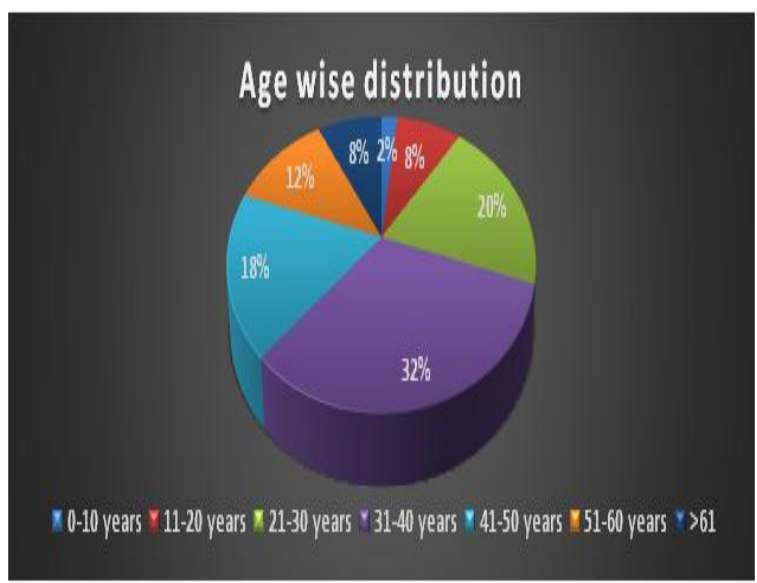

Table 2: Descriptive analysis of gender in study population $(\mathbf{N}=\mathbf{5 0})$

\begin{tabular}{|c|c|c|}
\hline Gender & No. of Patients & Percentage \\
\hline Male & 33 & $66.0 \%$ \\
\hline Female & 17 & $34.0 \%$ \\
\hline Total & $\mathbf{5 0}$ & $\mathbf{1 0 0 \%}$ \\
\hline
\end{tabular}

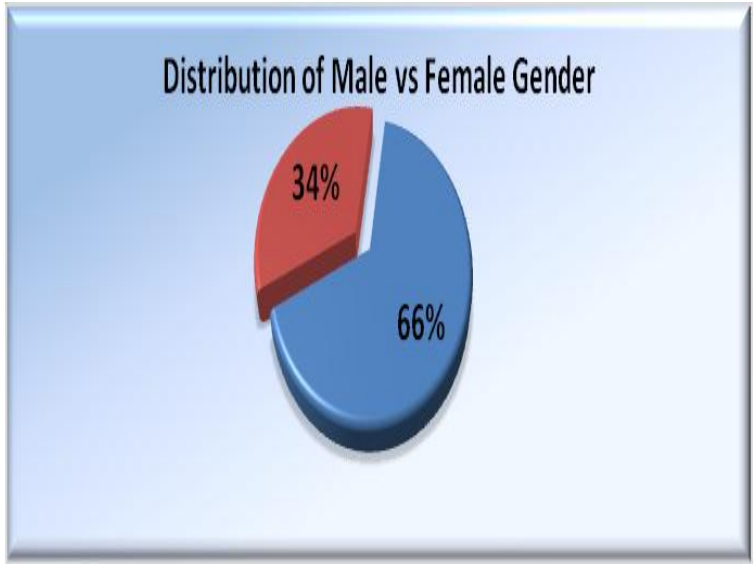

Table 3: Descriptive analysis of positive complains in study population $(\mathbf{N}=\mathbf{5 0})$

\begin{tabular}{|l|c|c|}
\hline \multicolumn{1}{|c|}{ Parameter } & No. Of Patients & Percentage \\
\hline Pain And Tenderness & 42 & $84.0 \%$ \\
\hline Stiffness & 26 & $52.0 \%$ \\
\hline Similar Past History & 9 & $18.0 \%$ \\
\hline Restriction of Movements & 14 & $28.0 \%$ \\
\hline Redness & 5 & $10.0 \%$ \\
\hline Old Trauma History & 8 & $16.0 \%$ \\
\hline
\end{tabular}

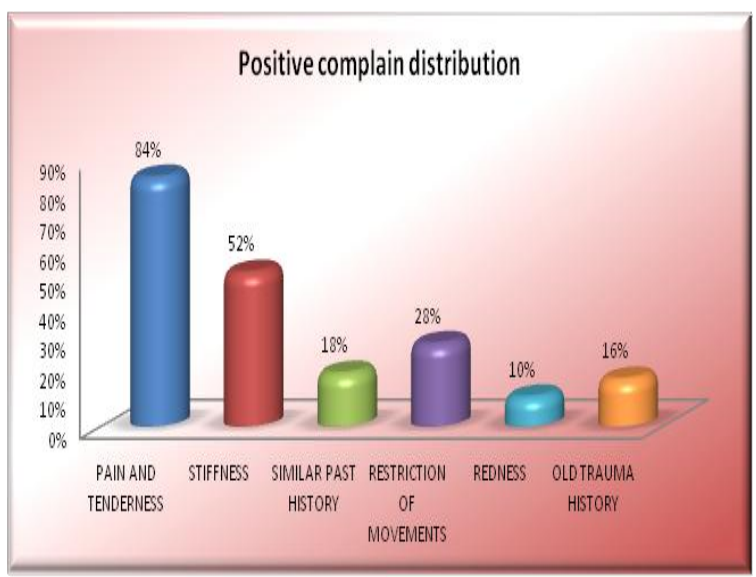

Table 5: Descriptive analysis of Lateralization of the patella in study population $(\mathrm{N}=50)$

\begin{tabular}{|c|c|c|}
\hline Lateralization of Patella & No. of Patients & Percentage \\
\hline Normal (Less Than 6 Mm) & 32 & $64.0 \%$ \\
\hline Abnormal (More Than 6 Mm) & 18 & $36.0 \%$ \\
\hline Total & $\mathbf{5 0}$ & $\mathbf{1 0 0 \%}$ \\
\hline
\end{tabular}

\section{LATERALIZATION OF PATELLA}

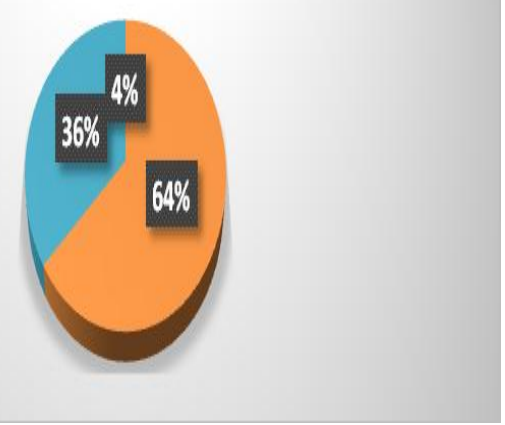


H.P Parekh et.al. Role of magnetic resonance imaging in study of the patellofemoral joint morphological abnormalities predisposing to patellar instability

Table 6: Descriptive analysis of Medial patellofemoral ligament (MPFL) injury

\begin{tabular}{|l|c|c|}
\hline $\begin{array}{c}\text { Medial Patello Femoral } \\
\text { Ligament Injuries }\end{array}$ & $\begin{array}{c}\text { No. of } \\
\text { Patients }\end{array}$ & Percentage \\
\hline Absent & 34 & $62.0 \%$ \\
\hline Complete Tear & 10 & $20.0 \%$ \\
\hline Partial Tear Total & 6 & $12.0 \%$ \\
\hline \multicolumn{1}{|c|}{ To } & $\mathbf{1 0 0}$ \\
\hline
\end{tabular}

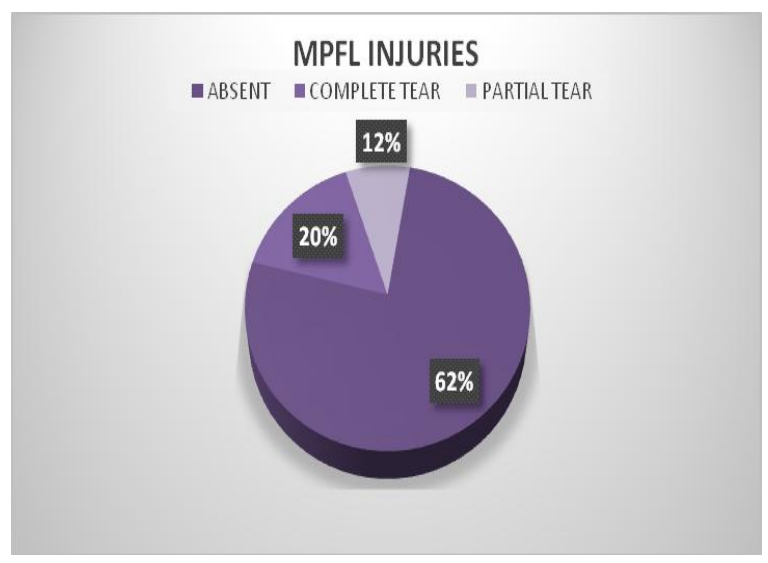

Table 4: Descriptive analysis of Trochlear measurements in the study population ( $N=50)$

\begin{tabular}{|l|c|c|}
\hline \multirow{2}{*}{} & \multicolumn{2}{|l|}{ Analysis of Trochlear Measurements } \\
\cline { 2 - 3 } & No. of Patients & Percentages \\
\hline Lateral Trochlear Inclination Angle (Degree) \\
\hline Abnormal or Decreased (11 or Below) & 5 & $10.0 \%$ \\
\hline Trochlear Facet Asymmetry (\%) & \multicolumn{2}{|}{} \\
\hline Increased/Abnormal (40 and Above) & 6 & $12.0 \%$ \\
\hline Trochlear DEPTH (mm) & 9 & $18.0 \%$ \\
\hline Abnormal or Decreased (Up to 2.9) & 9 & $18.0 \%$ \\
\hline Sulcal Angle & \multicolumn{2}{|l}{} \\
\hline Increased/Abnormal (144 and Above) & 9 & \\
\hline
\end{tabular}

Table 13: Descriptive analysis of patellar dislocation in study population $(\mathbf{N}=\mathbf{5 0})$

\begin{tabular}{|l|c|c|}
\hline MRI patellar dislocation & Frequency & Percentages \\
\hline Absent & 32 & $64.0 \%$ \\
\hline Lateral subluxation & 14 & $28.0 \%$ \\
\hline Prior / transient subluxation & 4 & $8.0 \%$ \\
\hline
\end{tabular}

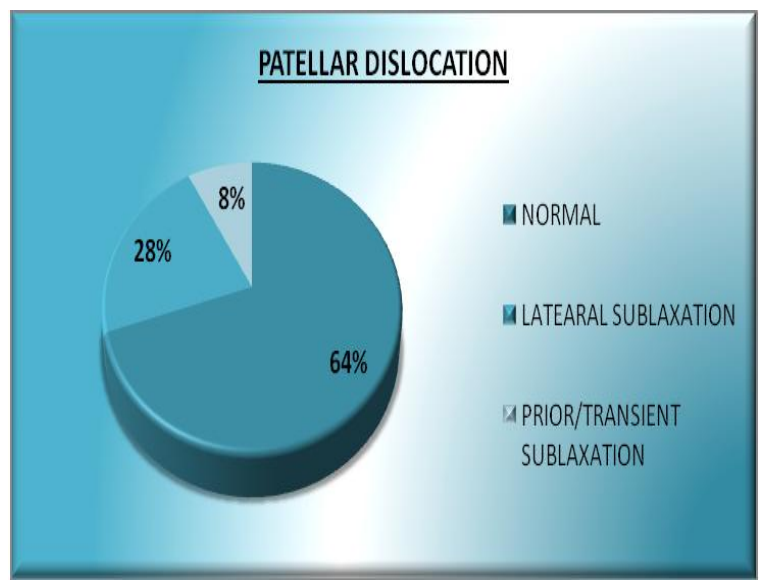

Table 7: Descriptive analysis of Insall-Salvatti index in study population $(\mathbf{N}=\mathbf{5 0})$.

\begin{tabular}{|l|c|c|}
\hline Insall-Salvatti Index & No. of Patients & Percentage \\
\hline Normal & 35 & $70.0 \%$ \\
\hline Patella Alta (1.3 and Above) & 8 & $16.0 \%$ \\
\hline Patella Baja (0.9 and Below) & 7 & $14.0 \%$ \\
\hline Total & $\mathbf{2 0}$ & $\mathbf{1 0 0 \%}$ \\
\hline \multicolumn{2}{|c|}{}
\end{tabular}

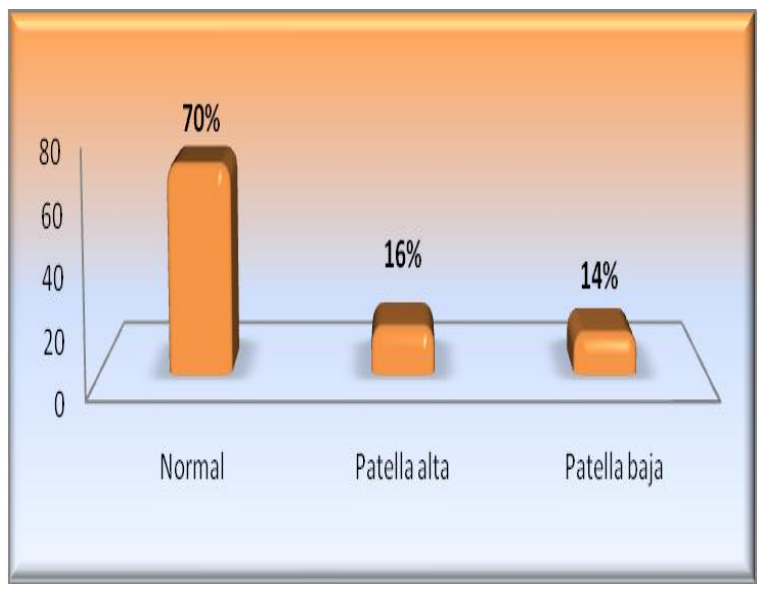

Table 8: Descriptive analysis of final diagnosis in the study population $(\mathbf{N}=50)$

\begin{tabular}{|l|c|c|}
\multicolumn{1}{|c|}{ Findings } & No. of Patients & Percentage \\
\hline Hoffa's Fat Pad Edema & 32 & $64.0 \%$ \\
\hline Chondromalacia Patella & 25 & $50.0 \%$ \\
\hline Sublaxated Patella & 18 & $36.0 \%$ \\
\hline Trochlear Dysplasia & 18 & $36.0 \%$ \\
\hline Mpfl Pathology & 16 & $32.0 \%$ \\
\hline Synovial Plica & 14 & $28.0 \%$ \\
\hline Synovial Hypertrophy & 9 & $18.0 \%$ \\
\hline Patella Ala & 8 & $16.0 \%$ \\
\hline Patella Baja & 7 & $14.0 \%$ \\
\hline Patellar Tendinosis & 6 & $12.0 \%$ \\
\hline Normal & 4 & $8.0 \%$ \\
\hline
\end{tabular}


H.P Parekh et.al. Role of magnetic resonance imaging in study of the patellofemoral joint morphological abnormalities predisposing to patellar instability

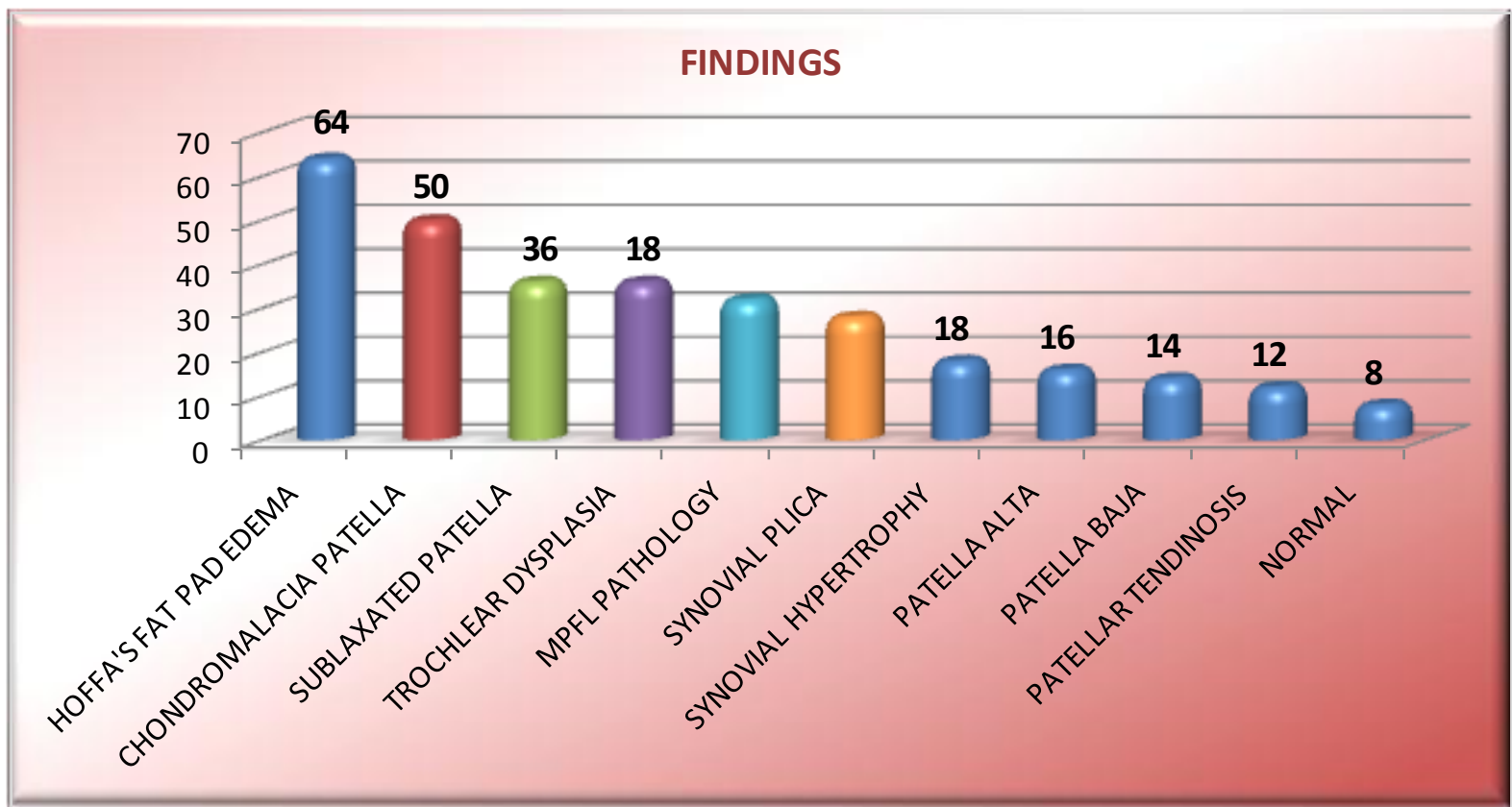

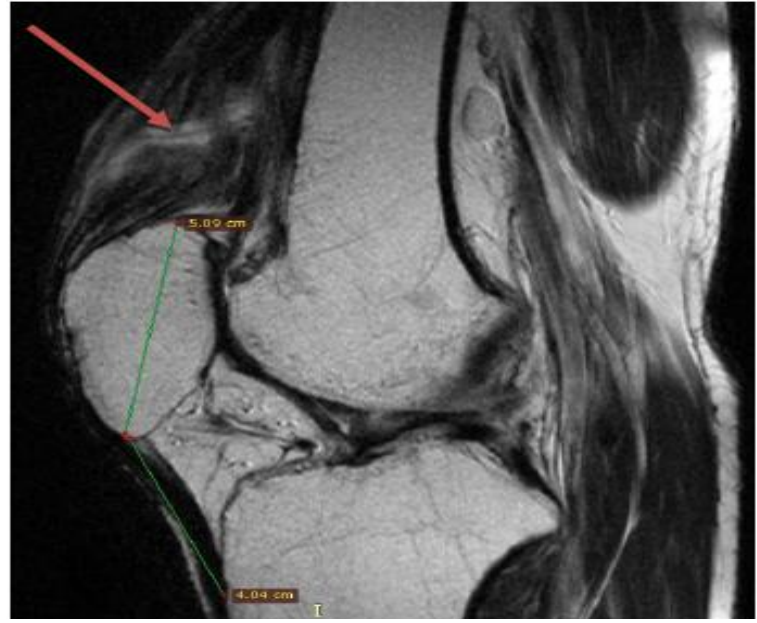

Figure 1: Above T1WI MR images show decreased Insall-Salvatti index $(0.79)$ in a patient with patella baja with quadriceps tendon tear (red arrow).

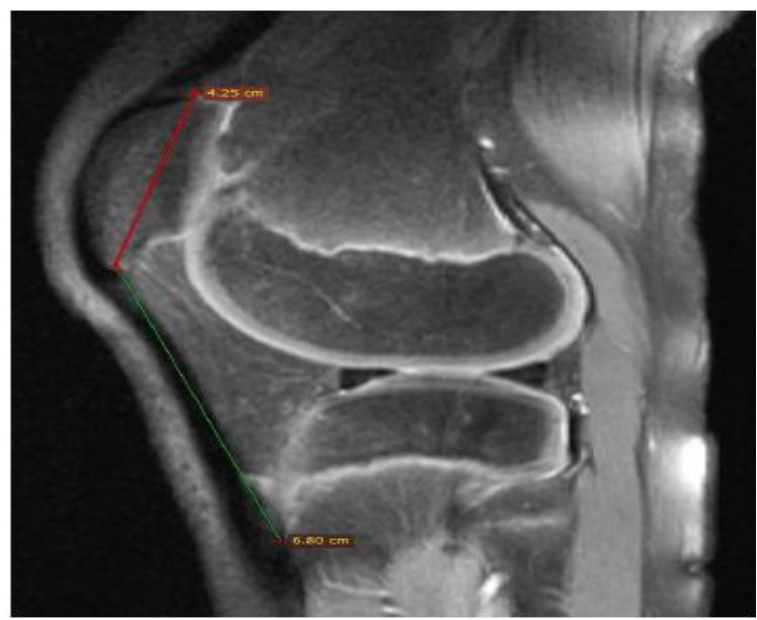

Figure 2: Above PD-FAT SAT MR images show increased InsallSalvatti index (1.35) in a patient with high riding patella or patella Alta.

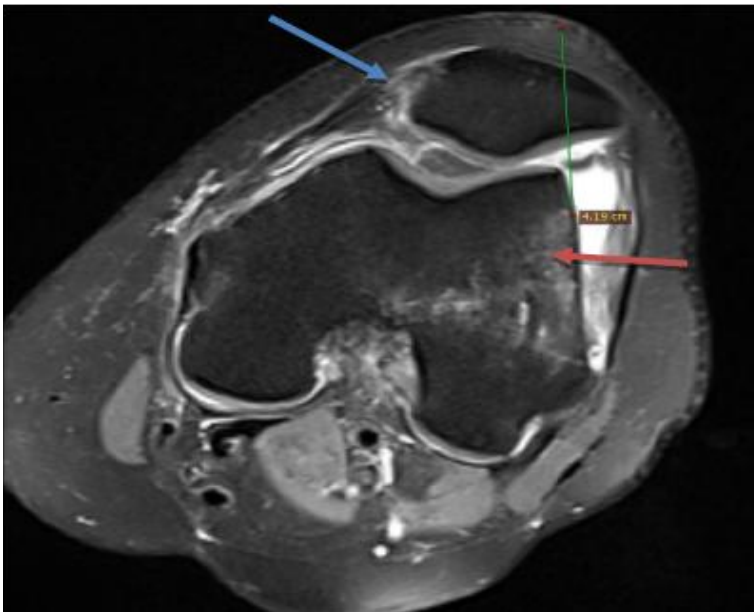

Figure 3: Above PD-FAT SAT MR images show MPFL tear (blue arrow) with lateralization of patella and bone contusion along medial patella and lateral femoral condyle (kissing contusion) (red arrow)

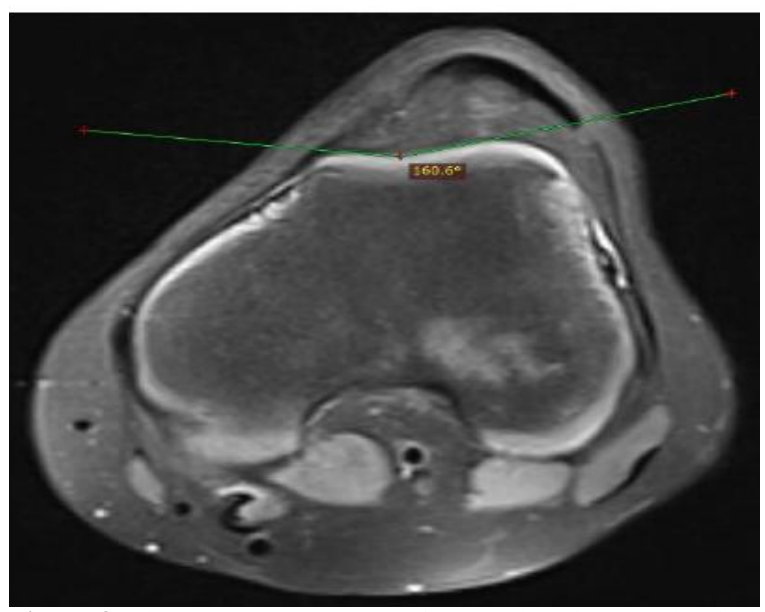

Figure 4: Above PD-FAT SAT MR images show increased Sulcal angle of 160 degrees (Trochlear dysplasia). 
H.P Parekh et.al. Role of magnetic resonance imaging in study of the patellofemoral joint morphological abnormalities predisposing to patellar instability

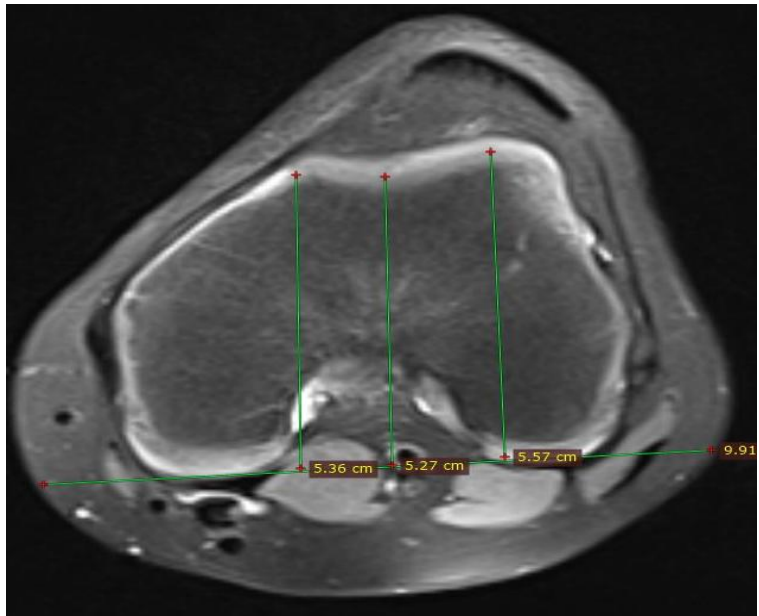

Figure 5: Above PD-FAT SAT MR images show shallow or decreased trochlear depth $(1.7 \mathrm{~mm})$ in case of trochlear dysplasia.

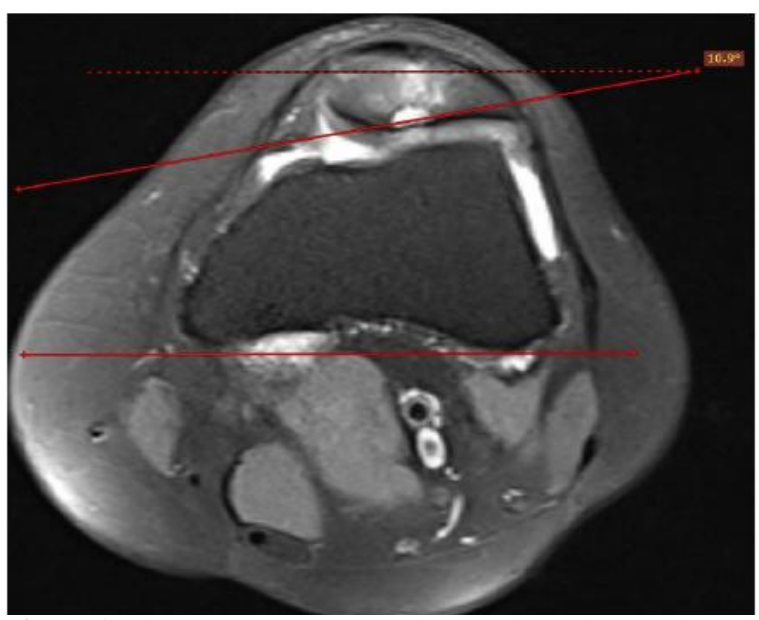

Figure 6: Above PD-FAT SAT MR images show abnormal lateral trochlear inclination angle (10.9 degree) in a case of trochlear dysplasia with Grade IV chondromalacia patella.

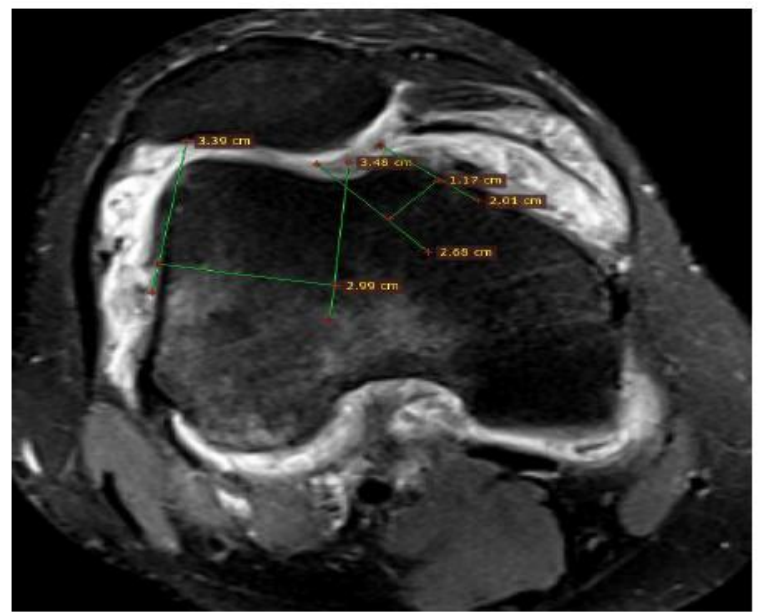

Figure 7: Above PD-FAT SAT MR images show abnormal trochlear facet asymmetry (37 degrees) is a trochlear dysplasia patient with lateralization of patella associated with MPFL tear and joint effusion.

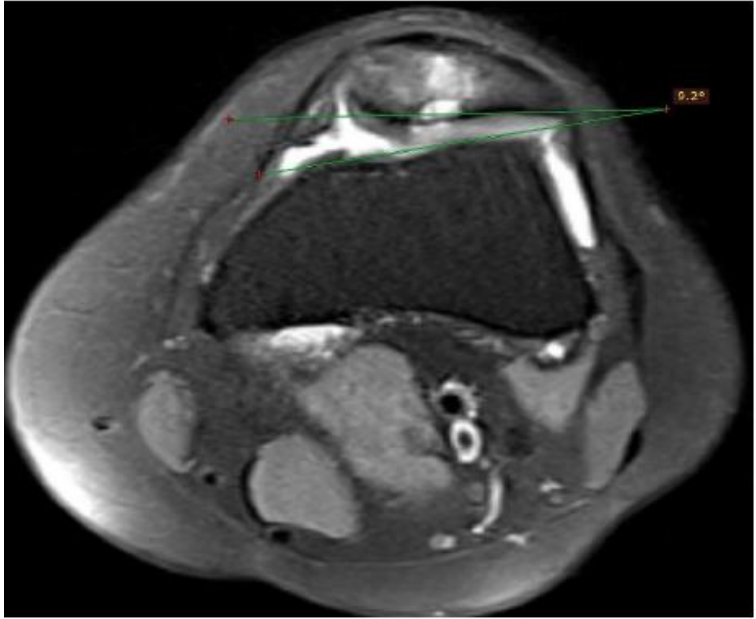

Figure 8: Above PD-FAT SAT MR images show abnormal patello-femoral angle (opening medially) in a case of dislocated patella with Grade IV chondromalacia patella

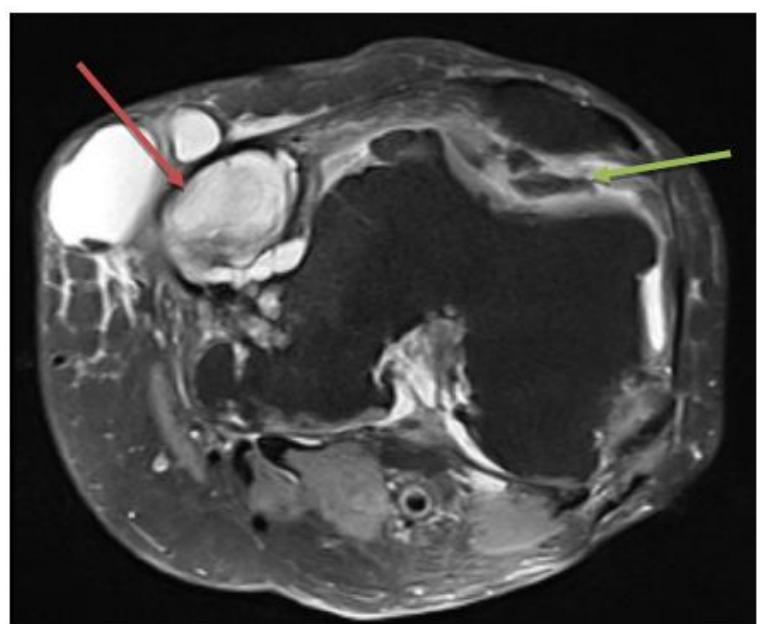

Figure 9: Above PD-FAT SAT MR images show complex ganglion cystic lesion (red arrow) along medial femoral condyle associated with MPFL tear with bone edema along medial condyle and degenerative changes with multiple osteophyte, loose bodies bone (green arrow) along joint space.

\section{DISCUSSION}

- In our study population included 50 subjects, age distribution in our study was in range of 8 years to 68 years with maximum population within 31- 40 years range. out of which $66 \%$ were males and $34 \%$ were females. Majority of the study population $84 \%$ had pain and tenderness while $52 \%$ presented with stiffness and $28 \%$ presented with restriction of movement.

- Patellofemoral stability is promoted by bone and soft-tissue structures. The soft tissues that restrict subluxation or lateral displacement of the patella include the medial patellofemoral ligament (MPFL), the medial patello-meniscal ligament, 
the medial patello-tibial. Patellofemoral instability is a common cause of knee pain and disability. Both Static and dynamic forces tend to displace the patella laterally, and in almost all cases. In our study, 18 (36\%) had subluxated or dislocated patella as confirmed by MRI. While in our study 16 (32\%) had MPFL tear out of which $10(20 \%)$ had complete MPFL tear and $6(12 \%)$ had a partial tear of MPFL and 34 (62\%) had no MPFL injury. The kissing contusion is that it tells about prior / transient patellar dislocation. In our study, kissing contusion was seen in $10(20 \%)$ of subjects. Balcarek $\mathrm{P}$ et al in their study observed that injury to the MPFL was found in $98.6 \%$ of the patients after the acute LPD, with a complete tear in $51.4 \%$, most commonly seen at the femoral attachment site and a partial tear in $48.6 \%$ (7). Their study showed that patterns of MPFL injury depend on trochlear dysplasia and patellar height. In our study, the correlation between MPFL injury and Patellar dislocation was strong. Patellar dislocation was present in $18(36 \%)$ and MPFL injury was seen in $16(32 \%)$ of subjects.

- Trochlear dysplasia has been identified as one of the main factors contributing to patello-femoral instability. In our study, trochlear dysplasia was observed in $18(36 \%)$ subjects, $9(18 \%)$ had shallow trochlear depth and increased Sulcal angle, $6(12 \%)$ subjects had abnormal or increased trochlear facet asymmetry and $5(10 \%)$ subjects had abnormal or decreased. In our study subluxated/dislocated patella was found in $18(36 \%)$ of subjects out of which 14 (22\%) had laterally subluxated dislocated patella and $4(8 \%)$ had prior / transient subluxated patella. Souza PM et al also reported that Trochlear Dysplasia was present in $51 \%$ of the cases who presented with patella: instability and were easily identified using MRI (8). The patellar height ratio known as the Insall-Salvatti index is a measure for Patella Alta/Baja. In our study, 35 (70\%) had normal InsallSalvatti index, 8 (16\%) had increased index (Patella Alta) and $7(14 \%)$ had patella baja.

- Chondromalacia patella is a relatively common condition characterized by fissuring within the articular cartilage of the patella and in severe cases, erosion of the cartilage and subchondral bone. Structural abnormalities, such as patella Alta, genu valgum and deformities of the femoral condyle ate believed to injure the articular surface of the patella because of patellar instability and increased stress on parts of the articular cartilage. In our study, $25(50 \%)$ of subjects were diagnosed with chondromalacia. Chondromalacia patellae was detected in 44 knees and was absent in 54 knees at arthroscopy in a study done by Endo $\mathrm{Y}$ et al (9). Patellar tendon pain is a frequent complaint in athletes who participate in jumping and running sports. In our study, 32 (64\%) had Hoffa's fat pad edema or impingement. Fahmy HS et observed in their study that in the 55 knees they analyzed for patellofemoral instability, them was Hoffa's fat pad edema in $55 \%$ of their study subjects (10).

\section{CONCLUSION}

The normal physiology of the patellofemoral joint (PFJ) is highly dependent on the congruent relationship of the patella with the femoral trochlear groove. Alterations in the surface geometry of the PFJ are associated with multiple clinical problems commonly patellofemoral instability (PFI) which leads to recurrent patellar dislocations. MRI is generally a safe, accurate, and specific modality which has emerged as the first modality of choice who present with anterior knee pain or in whom patellofemoral instability is clinically suspected. Also, it has a high specification for detecting the grades and the types of some of these diseases or factors that may 
predispose to them such as trochlear dysplasia and patella alta. MR imaging not only helps in diagnosing different anatomic variants, ligamentous injuries and chondral lesions that lead to instability but also guide surgeons in selecting the most optimal treatment.

\section{Acknowledgement: None}

\section{Conflict of Interest: None}

\section{Source of Funding: None}

\section{Ethical Approval: Approved}

\section{REFERENCES}

1. Kaplan EB. Some aspects of functional anatomy of the human knee joint. Clin Orthop. 1962;23(23):18-29.

2. Strachan WF. The knee joint applied anatomy. J Am Osteopath Assoc. 1954:53(11): 616-8.

3. Hungerford DS, Barry M. Biomechanics of the patellofemoral joint. Clin Orthop Relat Res. 1979(144):9-15.

4. Amis AA. Current concepts on anatomy and biomechanics of patellar stability. Sports Med Arthrosc. 2007; 15(2):48-56.

5. Salzmann GM, Weber TS, Spang JT, Imhoff AB, Schöttle PB. Comparison of native axial radiographs with axial $M R$ imaging for determination of the trochlear morphology in patients with trochlear dysplasia. Arch Orthop Trauma Surg. 2010; 130(3):335-40.

6. Khanna AJ, Cosgarea AJ, Mont MA, Andres BM, Domb BG, Evans P], et a1.
Magnetic resonance imaging of the knee. Current techniques and spectrum of disease. J Bone Joint Surg Am. 2001; 83-A Suppl 2 Pt 2:128-41.

7. Balcarek P, Ammon J , Frosch S, Walde TA, SchuttrumprP, Ferlemann KG, et a1. Magnetic resonance imaging characteristics of the medial patellofemoral ligament lesion in acute lateral patellar dislocations considering trochlear dysplasia, patella alta, and tibial tuberosity-trochlear groove distance. Arthroscopy. 2010;26(7):926-35.

8. Souza PM, Gabetto MS, Serrao MG, Vieira LA, De Oliveira DC. Patellofemora 'Instability: Evaluation by Magnetic Resonance Imaging. Rev Bras 0110p! 2013; 48(2):159-64.

9. End0 Y, Schweitzer ME, BordaloRodrigues M, Rokito AS, Babb JS. MRI quantitative morphologic analysis of patellofemoral region: lack of correlation with chondromalacia patellae at surgery. A] R Am J Roentgenol, 2007; 189(5):1 165-8.

10. Fahmy HS, Khater NH, Nasef NM, Nasef NM. Role of MRI in assessment of patellofemoral derangement in patients with anterior knee pain. The Egyptian journal of Radiology and Nuclear Medicine. 2016; 47(4): 1485-92.

How to cite this article: H.P Parekh, S Aravind Prasad Naik. Role of magnetic resonance imaging in study of the patellofemoral joint morphological abnormalities predisposing to patellar instability. International Journal of Science \& Healthcare Research. 2021; 6(2): 208-215. DOI: https://doi.org/10.52403/ijshr. 20210438 